
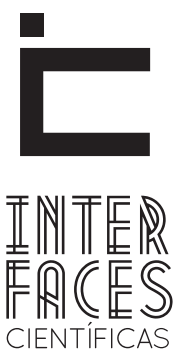

SAÚDE E AMBIENTE

\title{
PREVALÊNCIA DE LESÕES EPITELIAIS DO TRATO GENITAL INFERIOR EM ADOLESCENTES EM ARACAJU/SERGIPE
}

Meirelles Barros Santos Gustavo Teles Matos ${ }^{3}$
Jacqueline Mazzotti Cavalcanti Silva ${ }^{2}$ Júlia Maria Gonçalves Dias ${ }^{4}$

\section{RESUMO}

0 início precoce da atividade sexual e relacionamentos com múltiplos parceiros são fatores relevantes associados à carcinogênese cervical. Devido à diferença da anatomia topográfica da cérvice da adolescente da do adulto. 0 objetivo desta pesquisa é determinar a prevalência de lesões epiteliais do trato genital inferior em pacientes adolescentes atendidas no Centro de Atenção Integral á Saúde da Mulher. Os métodos aqui utilizados são: estudo observacional descritivo, com investigação de 35 pacientes adolescentes com alterações epiteliais no trato genital inferior, confirmadas por biópsias. As pacientes foram atendidas no Centro de Atenção Integral á Saúde da Mulher, em Aracaju, entre maio de 2012 e maio de 2013. Foram incluídas no estudo todas as pacientes que atendiam aos critérios de inclusão e aceitaram participar da pesquisa por meio de consentimento livre e esclarecido. Para análise estatística foi utilizado o programa EPI-INFO 7 e foram realizados testes de associação exato de Fisher e Mantel-Haenszel. A pesquisa foi submetida ao Comitê de Ética em Pesquisa Envolvendo Seres Humanos da UFS. Os resultados alcançados: em 35 adolescentes que participaram da pesquisa $85,71 \%$ apresentaram alterações sugestivas de infecção pelo papiloma vírus humano (HPV). Cinco pacientes, 14,29\%, apresentaram lesão de baixo grau e nenhuma adolescente apresentou lesão de alto grau. A média de idade foi em torno de 17,4 anos, a primeira relação sexual entre 12 e 14 anos e a maioria teve de 1 a 2 parceiros. Foi encontrada associação entre 0 diagnóstico da paciente e o uso de preservativo e associação bastante significativa entre o diagnóstico e o número de preventivos realizados.

\section{PALAVRAS-CHAVE}

Adolescentes. HPV. Neoplasia Intra-epitelial Cervical. Câncer de Colo. 


\section{ABSTRACT}

The beginning of sexual activity at an early age and relationships with multiple partners are relevant factors associated with cervical carcinogenesis due to the anatomical differences of the cervix between teenagers and adults. This study aimed to determine the prevalence of lower genital tract lesions in adolescent on treatment at the Women's Integrated Health Care Center. The work was a descriptive observational study in which were studied 35 adolescents with epithelial changes at the lower genital tract confirmed by biopsies. These patients were being treated at the Women's Integrated Health Care Center in Aracaju, between May 2012 and May 2013. This study covered all patients who met the inclusion criteria and agreed to participate through informed consent. For statistical analysis, the EPI-INFO 7 program was used and Fisher and Mantel-Haenszel exact tests were performed combination. This research was submitted to the Ethics Committee on Research Involving Human Subjects of the Federal University of Sergipe. In 35 adolescents who participated in the survey $85.71 \%$ had abnormalities suggestive of infection by human papilloma virus (HPV). Five patients, $14.29 \%$, had low-grade lesion and no teenager had a high-grade lesion. Conclusion: Conclusion: The mean age was around 17.4 years, the first intercourse between 12 and 14 years and most had 1-2 partners. Association was found between the diagnosis of the patient and the use of condoms and highly significant association between the diagnosis and the number of preventive conducted.

\section{KEYWORDS}

Adolescents. HPV. Cervical Intraepithelial Neoplasia. Cervical Cancer.

\section{RESUMEN}

La precocidad la actividad sexual es un factor relevante asociado a la carcinogénesis cervical debido a la diferencia de la anatomía topográfica del cuello uterino de la adolescente y de la mujer adulta. El objetivo fue determinar la prevalencia de las lesiones del tracto genital inferior en pacientes adolescentes tratadas en el Centro de Atención Integral a la Salud de la Mujer. Fue un estudio observacional descriptivo que investigó 35 pacientes adolescentes con lesiones epiteliales en el tracto genital inferior confirmadas por biopsia. Las pacientes fueron tratadas en el Centro de Atención Integral a la Salud de la Mujer en Aracaju entre mayo de 2012 y mayo de 2013. Eso estudio incluyó todas las pacientes que cumplieron los criterios de inclusión y aceptaron participar por el consentimiento informado. El análisis estadístico se realizó utilizando el EPI-INFO 7 y pruebas de asociación como la prueba exacta de Fisher y de
Mantel- Haenszel. El estudio fue presentado al Comité de Ética en Investigación en Seres Humanos de la Universidad Federal de Sergipe. De las 35 adolescentes que participaron de la encuesta $85,71 \%$ fueron diagnosticadas con papiloma virus humanas (VPH). Cinco pacientes, 14,29\%, tenían lesiones de bajo grado y ninguna tuvo lesión de alto grado. Conclusión: las pacientes tenían cerca de 17,4 años y la mayoría tuvo su primera relación sexual entre los 12 y los 14 años y tuvo 1-2 parejas. Se encontró asociación entre el diagnóstico del paciente y el uso del preservativo y, también, asociación entre el diagnóstico y el número de consultas preventivos realizadas.

\section{PALABRAS CLAVE}

Adolescentes. HPV. Neoplasia intraepitelial cervical. Cáncer de cuello uterino. 


\section{INTRODUCC̃̃O}

A adolescência é fase de transição entre a infância e a idade adulta, e como fase própria, possui características singulares nos campos biológicos, psicológicos e sociais (SCHOEN-FERREIRA, 2010).

A adolescência é um período importante para exploração e desenvolvimento sexual. Durante este tempo as pessoas começam a considerar raros os comportamentos sexuais de acordo com a sua preferência e a sua faixa etária (COLLINS, 2004).

Estudos sobre o início da vida sexual e o uso de contraceptivos e preservativos têm indicado que adolescentes e jovens tendem a não usá-los quando: iniciam a vida sexual muito cedo e definem a relação em que ocorreu sua iniciação sexual como casual; no caso de adolescentes do sexo feminino, quando têm parceiros mais velhos (mais de sete anos) ou de outra geração (PAIVA et al., 2008).

Apesar da importância do tema para a formação integral da adolescente, existem poucos estudos no Brasil a esse respeito e, na prática diária da profissão, os pediatras estão pouco afeitos a tratar da questão da sexualidade. Por outro lado, nesta época aflora a temática da sexualidade (VITALLE, 2003).

As doenças sexualmente transmissíveis (DSTs) constituem-se ainda um sério problema de saúde pública, principalmente na adolescência, podendo deixar sequelas, curáveis ou não, como infertilidade, gravidez ectópica, câncer genital, doença hepática crônica, entre outras (ROMERO et al., 2007).

A inclusão da educação sexual nas escolas contribui para postergar a iniciação sexual e não há evidências de que o ensino estimule a adolescente a ter relações sexuais (HASSAN-CREATSAS, 2002).
A alta vulnerabilidade das adolescentes ao HPV é atribuída à maior exposição da zona de transformação da cérvice, junção escamocolunar (JEC), durante a adolescência do que na vida adulta (LONGATTO FILHO et al., 2003). As infecções por HPV de alto risco em mucosassão associados com uma variedade decâncer, especialmente do câncer do colo do útero, que são quase uniformemente causadas por HPV de alto risco (MCLAUGHLIN-DRUBIN; MÜNGER, 2009).

Alguns estudos mostram que a idade da primeira atividade sexual (menos de 18 anos de idade), continua a ser um fator de risco significativo para o eventual desenvolvimento de câncer cervical (MOSCICKI, 2008).

Aspectos sociodemográficos, comportamentais, sexuais, contraceptivos, reprodutivos e/ou clínicos tornam a mulher mais susceptível a outros fatores mais diretamente envolvidos na carcinogênese cervical, tais como inflamação local e infecção por HPV (PINTO; FUZII; QUARESMA, 2011).

O HPV é adquirido por meio de relações sexuais, mas a maioria dos HPVs sofre uma espécie de clearance, no prazo de dois anos na maioria das mulheres. Uma minoria de infecções por HPV podem persistir, e indivíduos com infecção por HPV de alto risco persistente tem um risco substancial de desenvolvimento de pré-câncer cervical, ou Neoplasia Intra Epitelial Cervical III (NIC). As NICs III são os alvos de rastreio, porque mais de um terço deles irá evoluir para o câncer cervical invasivo dentro de 10-20 anos (GRAVITT, 2011).

Anualmente, cerca de 5-15\% das mulheres previamente sem o HPV (são infectadas com qualquer tipo de HPV de alto risco e aproximadamente $25 \%$ da incidência da infecção se concentram na faixa etária dos 15-19 anos) (SELLORS, 2003). 
Um dos maiores objetivos a serem alcançados pelo profissional é conseguir que a adolescente se sinta responsável pela sua própria saúde, de forma integral. Deve-se tentar compreender a dificuldade que a adolescente tem em verbalizar suas dúvidas e inquietudes (VENTURA, 2003).

Segundo o instituto nacional do câncer, o método de rastreamento do câncer do colo do útero no Brasil é o exame citopatológico (exame de Papanicolaou), que deve ser oferecido às mulheres na faixa etária de 25 a 64 anos e que já tiveram atividade sexual. É importante destacar que a priorização de uma faixa etária não significa a impossibilidade da oferta do exame para as mulheres mais jovens ou mais velhas. Na prática assistencial, a anamnese bem realizada e a escuta atenta para reconhecimento dos fatores de risco envolvidos e do histórico assistencial da mulher são fundamentais para a indicação do exame de rastreamento.

Desse modo, este estudo tem como objetivo determinar a prevalência de infecções por HPV e de alterações epiteliais cervicais em adolescentes atendidas no Centro de Atenção Integral à Saúde da Mulher (CAISM) e caracterizar epidemiologicamente esta população do ponto de vista sociodemográfico e sociocomportamental.

\section{MÉTODO}

Foi realizado um estudo do tipo observacional descritivo. A amostra incluiu 35 pacientes adolescentes do sexo feminino com alterações epiteliais no trato genital inferior e/ou sugestão de infecção por HPV confirmadas por biópsias com idades entre 13 e 19 anos que foram atendidas no Centro de Atenção Integral à Saúde da MuIher, no período de maio de 2012 a maio de 2013. Todas as entrevistadas foram informadas pelos pesquisadores dos reais motivos da pesquisa bem como da preservação de suas identidades, por meio da leitura e assinatura do Termo de Consentimento Livre e Esclarecido.
Foram excluídas da amostra as pacientes que não aceitaram participar da pesquisa, se negaram a assinar o termo de consentimento livre esclarecido ou vinham de outros estados.

O local de coleta de dados e de realização do estudo foi CAISM, referência estadual para o atendimento feminino, apresentando o perfil de atendimento em média complexidade referente às lesões precursoras do câncer uterino e de mama e assistência ao pré-natal de alto risco. O CAISM engloba os serviços de patologia cervical e uterina, mastologia, citopatologia, pré-natal de alto risco, ultrassonografia, assistência social e enfermagem.

A coleta de dados foi realizada mediante entrevista feita diretamente à paciente, a partir de um questionário fechado e direcionado ao tema central. Foram colhidos dados pessoais gerais da paciente, sem, contudo, identificá-la, como procedência, idade, escolaridade, convívio com os pais, início de vida sexual, número de parceiros, estado civil, uso de condom, uso de outros contraceptivos, número de gestações, de paridade e de abortos, número de preventivos realizados, antecedentes de DST e diagnóstico.

Após digitação dos dados foi utilizado para a análise estatística o software, de domínio público, EPI-INFO 7 e para demonstração das variáveis foram elaboradas tabelas de distribuição de frequência e elaboradas tabelas de associação de variáveis utilizando o testes exato de Fisher e de Mantel-Haenszelcom valor de $p \leq 0,05$. Não foi realizado cálculo amostral, pois a amostra foi composta por todas as pacientes atendidas no serviço no período do estudo.

A pesquisa foi submetida ao Comitê de Ética da Universidade Federal de Sergipe, cujo protocolo foi CAAE 09947513.8.0000.5546. 


\section{RESULTADOS}

A idade das pacientes variou de 13 a 19 anos, havendo predomínio de dois grupos analisados, entre 15 e 17 anos e entre 18 e 20 anos, com porcentagens iguais de $45,72 \%$ para esses dois grupos. No estudo não houve pacientes analfabetas, a maioria das pacientes, $65,71 \%$ cursavam o ensino médio. Quanto à procedência, $22(62,86 \%)$ das adolescentes que participaram da pesquisa eram do interior, $25(71,43 \%)$ afirmaram ainda morar com os pais e 10 $(28,57 \%)$ moram sem estes. Com relação ao estado civil, 28 adolescentes (80\%) disseram ser solteiras.

Tabela 1 - Distribuição das frequências dos dados sócio-demográficos das adolescentes atendidas

\begin{tabular}{lcc}
\hline Variáveis & Número Frequência & Valor de p \\
\hline Idade & & \\
$12-14$ anos & 03 & $8,6 \%$ \\
$15-17$ anos & 16 & $45,7 \%$ \\
$18-20$ anos & 16 & $45,7 \%$ \\
Escolaridade & & $p=0,639$ \\
Ens. Fund. & 11 & $31,4 \%$ \\
Ens. Méd. & 23 & $65,7 \%$ \\
Ens. Sup. & 01 & $2,9 \%$ \\
Procedência & & $p=0,052$ \\
Aracaju & 13 & $37,1 \%$ \\
Interior & 22 & $62,9 \%$ \\
Convívio com os pais & & $p=0,291$ \\
Sim & 25 & $71,4 \%$ \\
Não & 10 & $28,6 \%$ \\
Estado civil & & \\
Solteira & 28 & $80,0 \%$ \\
Solteira/União estável & 05 & $14,3 \%$ \\
Casada & 02 & $5,7 \%$ \\
\hline Foste: &
\end{tabular}

Fonte: Dados da pesquisa.

Dezenove pacientes $(54,2 \%)$ afirmaram usar preservativos ocasionalmente e 12 (34,2\%) afirmaram não usar. Vinte e oito pacientes eram nuligestas e somente uma $(2,8 \%)$ referiu ter sofrido aborto, sendo este espontâneo. Quando indagadas sobre o número de preventivos realizados durante toda a vida, 24 pacientes $(68,3 \%)$ afirmaram já terem sido submetidas ao preventivo de $1 \mathrm{a}$ 3 vezes durante sua vida e 4 pacientes $(11,4 \%$ ) referiram nunca terem sido submetidas a esse exame. Entre todas as pacientes, somente uma $(2,9 \%)$ afirmou ter antecedente de DST, sendo esta sífilis. 0 teste exato de Fischer encontrado para a relação entre a variável início da vida sexual e diagnóstico foi de 0,346 .

Tabela 2 - Distribuição de frequência dos dados sexuais das adolescentes atendidas

Variav

Início da vida sexual

Número

Frequência

\begin{tabular}{lcr}
\hline $12-14$ anos & 16 & $45,7 \%$ \\
$15-17$ anos & 14 & $40 \%$ \\
18-20 anos & 05 & $14,3 \%$ \\
Número de parceiros & & \\
$1-2$ & 23 & $65,7 \%$ \\
$3-4$ & 08 & $22,9 \%$ \\
$5-6$ & 02 & $5,7 \%$ \\
Mais de 6 & 02 & $5,7 \%$ \\
Uso de Condom & & \\
Sim & 04 & $11,4 \%$ \\
Ocasional & 19 & $54,3 \%$ \\
Não & 12 & $34,3 \%$ \\
Gestações & & \\
00 & 28 & $80,0 \%$ \\
01 & 04 & $11,4 \%$ \\
02 ou mais & 03 & $8,6 \%$ \\
Partos & & \\
00 & 29 & $82,9 \%$ \\
01 & 04 & $11,4 \%$ \\
02 ou mais & 02 & $5,7 \%$ \\
Abortos & & \\
00 & 34 & $97,1 \%$ \\
01 & 01 & $2,9 \%$ \\
No de preventivos realizados & \\
00 & 04 & $11,4 \%$ \\
$1-3$ & 24 & $68,3 \%$ \\
$4-7$ & 04 & $11,4 \%$ \\
Mais de 7 & 03 & $8,6 \%$ \\
Antecedentes de DST & & \\
Sim & 01 & $97,9 \%$ \\
Não & 34 & \\
\hline Fonte: Dados da pesquisa. & & \\
\hline & &
\end{tabular}


Das 35 pacientes que participaram da pesquisa, 30 pacientes $(85,7 \%)$ foram diagnosticadas com condiloma (HPV). As cinco pacientes restantes (14,3\%) tinham o diagnóstico de lesão de baixo grau por NIC I, não sendo nenhuma paciente diagnosticada com NIC II ou NIC III (gráfico 01).

Gráfico 1 - Prevalência das lesões epiteliais do trato genital inferior nas pacientes

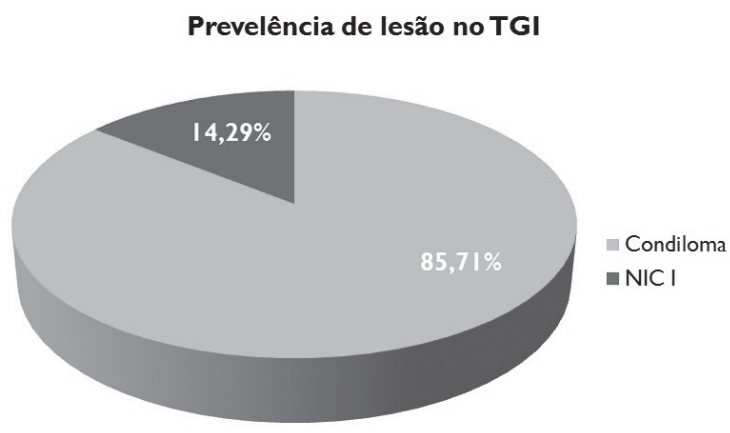

Fonte: Dados da pesquisa.

Houve associação entre o diagnóstico da paciente e o número de preventivos realizados, $p=0,0289$. Todas as pacientes diagnosticadas com NIC I ou nunca foram submetidas a exame preventivo ou só o fizeram uma vez durante toda sua vida. Dentre as pacientes que apresentaram alterações sugestivas de infecção por HPV, 46,7\% nunca realizaram ou só fizeram o preventivo uma vez e $53,3 \%$ já o fizeram mais de uma vez.

Também houve associação entre o diagnóstico e o uso de condom, $p=0,00$. Entre as pacientes diagnosticadas com condilomas (HPV), 96,7\% disseram não fazer uso do preservativo. Já entre as pacientes diagnosticadas com NIC I a ausência do uso de preservativo cai para $40 \%$. Não houve associação entre o início da vida sexual e o tipo de diagnóstico $(p=0,569)$. Entre as pacientes diagnosticadas com condilomatose, metade destas tinha iniciado a vida sexual antes dos 15 anos e a outra metade depois dos 15 anos.

Das cinco pacientes diagnosticadas com NIC I, $20 \%$ tinham iniciado a vida sexual antes dos 15 anos e $80 \%$ depois. Não foi encontrada associação entre o número de parceiros e o diagnóstico da paciente ( $p=$ $0,569)$. Trinta pacientes foram diagnosticadas com condilomas, sendo que $66,7 \%$ destas tiveram de um a dois parceiros e $33,3 \%$ tiveram mais de dois parceiros. Das cinco pacientes que tinham o diagnóstico de NIC I, $60 \%$ tiveram de um a dois parceiros e $40 \%$ tinham mais de dois parceiros (tabela 03).

Tabela 3 - Associação entre o tipo de diagnóstico e o número de preventivos realizados pela paciente adolescente atendida no Centro de Atenção Integral à Saúde da Mulher

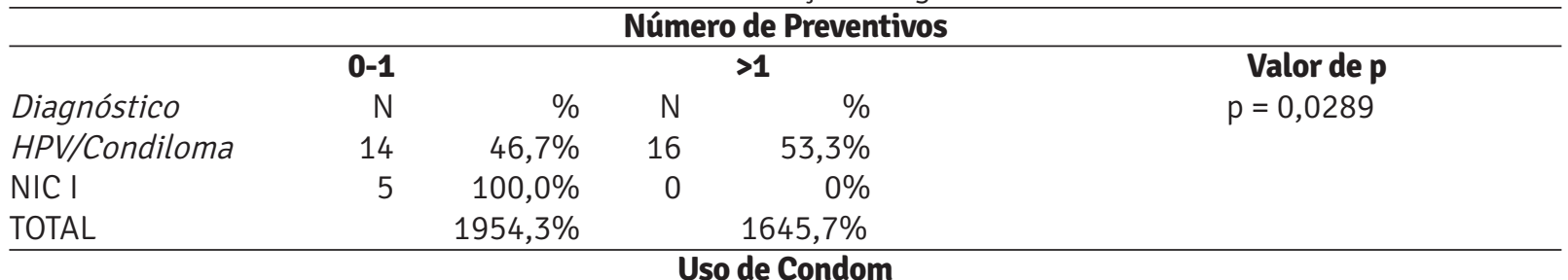

\begin{tabular}{lrrrrr}
\hline & Não & & \multicolumn{1}{c}{ Sim } & Valor de p \\
Diagnóstico & $\mathrm{N}$ & $\%$ & $\mathrm{~N}$ & $\%$ & $\mathrm{p}=0,000$ \\
HPV/Condiloma & 29 & $96,7 \%$ & 01 & $3,3 \%$ & \\
NIC I & 02 & $40,0 \%$ & 03 & $60,0 \%$ & \\
TOTAL & 31 & $88,6 \%$ & 04 & $11,4 \%$ &
\end{tabular}




\begin{tabular}{|c|c|c|c|c|c|c|c|c|}
\hline \multicolumn{9}{|c|}{ Início da vida sexual } \\
\hline & \multicolumn{2}{|c|}{ Menor de 15 anos } & \multicolumn{3}{|c|}{ Maior igual a 15 anos } & & & \multirow{2}{*}{$\begin{array}{l}\text { Valor de } \\
p=0,569\end{array}$} \\
\hline Diagnóstico & $\mathrm{N}$ & $\%$ & $\mathrm{~N}$ & \multicolumn{2}{|c|}{$\%$} & & & \\
\hline HPV/Condiloma & 15 & $50,0 \%$ & 15 & \multicolumn{2}{|c|}{$50,0 \%$} & & & \\
\hline $\mathrm{NICI}$ & 1 & $20,0 \%$ & 4 & \multicolumn{2}{|c|}{$80,0 \%$} & & & \\
\hline TOTAL & 16 & $45,7 \%$ & 19 & \multicolumn{2}{|c|}{$54,3 \%$} & & & \\
\hline \multicolumn{9}{|c|}{ Número de parceiros } \\
\hline & \multicolumn{2}{|c|}{ Menor igual a 2} & & \multicolumn{2}{|c|}{ Mais de 2} & Tota & & Valor de $\mathrm{p}$ \\
\hline Diagnóstico & $\mathrm{N}$ & $\%$ & & $\mathrm{~N}$ & $\%$ & $\mathrm{~N}$ & $\%$ & $p=0,569$ \\
\hline HPV/Condiloma & 20 & $66,7 \%$ & & 10 & $33,3 \%$ & & $30 \%$ & \\
\hline NIC I & 3 & $60,0 \%$ & & 2 & $40,0 \%$ & & $5 \%$ & \\
\hline TOTAL & 23 & $65,7 \%$ & & & $1234,3 \%$ & & 35 & \\
\hline
\end{tabular}

\section{DISCUSSÃO}

A maioria das adolescentes entrevistadas eram maiores de 15 anos $(91,4 \%)$ sendo a média de idade encontrada de 17,4 anos (desvio padrão de 6,13) para as pacientes estudadas. Em um estudo realizado em Pelotas com 960 pacientes, foi encontrada uma média semelhante de 17,7 anos (CRUZEIRO, 2010). Outro estudo realizado em Atlanta encontrou que $80 \%$ das pacientes tinham entre 14 e 16 anos (TARKOWISKI, 2010).

Foi constatado que jovens com baixa escolaridade tendem a iniciar sexualmente mais cedo do que as adolescentes com maior escolaridade. No presente estudo todas as pacientes eram alfabetizadas sendo que $65,7 \%$ delas estavam cursando o ensino médio e 31,4\% estavam cursando o ensino fundamental (WELLINGS, 2006).

O presente estudo observou $45,7 \%$ das adolescentes tiveram sua primeira relação sexual mais precocemente, sendo a média de idade entre 12 e 14 anos. Já o estudo realizado pela Faculdade de Saúde Pública da Universidade de São Paulo, com 5040 adolescentes, observou adolescentes com idade média de início da vida sexual mais tardiamente sendo de 14,9 anos (PAIVA, 2008). Como também a análise realizada em 1664 adolescentes pelo Instituto de Saúde Coletiva da UFBA, que encontrou a média de idade da primeira relação sexual das adolescentes de 15 anos (ALMEIDA, 2003).
Vinte por cento das adolescentes que participaram dessa pesquisa referiram gravidez, porém, apenas $17,1 \%$ referiram parto, já que houve um aborto espontâneo, correspondendo a 2,9\%. Em um estudo com 246 adolescentes de um reformatório em Mississipi, encontraram números semelhantes com $28 \%$ de gestação, $24 \%$ de parto e $4 \%$ de abortamentos (ROBERTSON, 2011). Um estudo com 3137 realizado na cidade de Providence, nos Estados Unidos observou em seu estudo apenas $8 \%$ de gravidez e todas as pacientes negaram aborto (CLEMENTS, 2011).

Neste estudo, $65,7 \%$ das pacientes tiveram entre um e dois parceiros sexuais, semelhante a estudo realizado pelo Ministério da Saúde com 6006 pacientes, onde as adolescentes tiveram menos chances de ter dois ou três parceiros (SZWARCWALD, 2004).

Entre as adolescentes entrevistadas $34,28 \%$ negaram fazer uso do preservativo e $54,28 \%$ disseram fazer uso deste ocasionalmente. Uma hipótese provável para a alta prevalência pode ser a não aceitação do preservativo pelo parceiro. Em um estudo realizado na Universidade do Paraná com 967 jovens, foi encontrado em seu estudo resultados que corroboram com essa hipótese em que 38\% das adolescentes referiram não usar preservativo (MOSER, 2007). Já no 
estudo realizado em Atlanta, foram encontrados valores inferiores onde $22 \%$ das pacientes afirmaram não fazer uso de preservativo (TARKOWISKI, 2010).

Apenas $2,86 \%$ das pacientes entrevistadas informaram quanto à presença de DST, sendo prevalente a sífilis, discordando com o estudo feito em uma escola do sul de São Paulo com 134 adolescentes, que observaram $27,6 \%$ das pacientes com história prévia de DST (CIRINO, 2010). Já no estudo com 246 adolescentes de um reformatório em Mississipi, foi encontrada uma prevalência de $19 \%$ de DSTs em sua pesquisa, porém a sífilis foi a mais prevalente com $40 \%$ assim como em nosso estudo (ROBERTSON, 2011).

Houve associação entre o tipo de diagnóstico e o uso de condom, e entre o diagnóstico e o número de preventivos realizados, provavelmente a ausência do uso do preservativo como fator de risco para a aquisição da infecção por HPV contribuiu para essa associação já que entre as pacientes que foram diagnosticadas com HPV, $96 \%$ não faziam uso de preservativo.

Com relação à associação significativa com o número de preventivos podemos observar que embora $46 \%$ das pacientes nunca tenham realizado o exame preventivo ou feito apenas uma vez. A realização do exame preventivo mesmo que seja uma única vez se mostra uma ferramenta eficaz no diagnóstico de lesões epiteliais HPV induzidas em adolescentes.

Entre as pacientes, 14,2\% foram diagnosticadas com NIC I e 85,7\% com condilomas. Em um estudo realizado na Finlândia entre os anos de 1997 e 2009 com 20.011 mulheres, encontraram 51,0\% de diagnóstico isolado de lesões sugestivas de HPV, achados inferiores ao nosso estudo (JACKOBSSON, 2013). Outro estudo realizado em São Francisco, Califórnia, encontrou prevalência em torno de 53\% de diagnóstico de lesões de baixo grau, achados superiores ao presente estudo (MOSCICKI, 2008).
0 achado de lesões de baixo grau foi significativo, embora saibamos que entre adolescentes essas lesões podem em sua maioria regredir espontaneamente, porém em virtude do alto índice de lesões condilomatosas encontradas e do baixo uso de métodos de barreira entre as entrevistadas, há uma evidência favorável para que o exame preventivo seja realizado nestas pacientes já que se mostrou como uma ferramenta de diagnóstico e, também, de prevenção para lesões mais graves.

\section{CONCLUSÃO}

Neste estudo, concluiu-se que a maioria das pacientes entrevistadas tinha em torno de 17 anos e estava cursando o ensino médio, não havendo paciente analfabeta. Mais da metade das pacientes entrevistadas eram provenientes do interior e ainda moravam com os pais. Houve maior prevalência de adolescentes solteiras. Pouco menos da metade iniciou sua vida sexual entre 12 e 14 anos e mais da metade teve de um a dois parceiros. Mais da metade afirmou não fazer uso frequente do preservativo. A gravidez foi pouco prevalente e somente uma paciente referiu ter abortado, sendo este espontâneo.

Observou-se uma prevalência maior de pacientes que já foram submetidas ao exame preventivo e uma baixa prevalência de antecedentes de DSTs. 0 diagnóstico de lesões condilomatosas foi o mais frequente. Encontrou-se associação significativa entre o diagnóstico da paciente e o uso de preservativo, e entre o diagnóstico e o número de preventivos realizados.

\section{REFERÊNCIAS}

ALMEIDA, M. C.; AQUINO, E. M.; GAFFIKIN, L.; MAGNANI, R. J. Uso de contracepção por adolescentes de escolas públicas na Bahia. Revista de Saúde Pública, São Paulo, n.37(5), p.566-575, mai. 2003.

ASNAR-FARIAS, Maria; SCHOEN-FERREIRA, Teresa. Adolescência através dos séculos. Psicologia Teoria Pesquisa, Brasília, n.26, p.227-234, abr-jun. 2010. 
BRASIL. Ministério da Saúde. Secretaria de Atenção à Saúde. Departamento de Atenção Básica. Rastreamento (Série A: Normas e Manuais Técnicos. Cadernos de Atenção Primária n.29), Brasília, 2010.

CIRINO, F. M. S. B.; NICHIATA, L. Y. I.; BORGES, A. L. V. Conhecimento, atitude, práticas na prevenção do câncer. Escola Anna Nery Revista de Enfermagem, Rio de Janeiro, n.14 (1), p.126-134, jan-mar. 2010.

CLEMENTS, A. E.; et all. Prevalence and patient characteristics associated with CIN 3 in adolescents. American Journal of Obstetrics and Gynecology, Philadelphia, Caderno, p.1-7, 2011.

COLLINS R. L.; ELLIOTT, M. N.; BERRY, S. H.; KANOUSE, D. E.; KUNKEL, D.; HUNTER, S. B.; MIU, A. Watching Sex on Television Predicts Adolescent Initiation of Sexual Behavior. Pediatrics, Illinois, Caderno, p.280-289, 1 set. 2004.

CRUZEIRO, A. L. S.; SOUZA, L. D. M.; SILVA, R. A.; PINHEIRO, R. T.; ROCHA, C. L. A.; HORTA, B. L. Comportamento sexual de risco: fatores associados ao número de parceiros sexuais e ao uso de preservativo em adolescentes. Ciência e Saúde Coletiva, Rio de Janeiro, n.15, p.1149-1158, 2010.

GRAVITT P. E. The know unknowns of HPV natural history. The journal of Clinical Investigation, Durham, Caderno, p.4593-4599, 2011.

HASSAN, E. A; CREATSAS, G. C. Adolescent sexuality: A developmental milestone or risk: taking behavior? The role of health care in the prevention of sexually transmitted diseases. Journal of Pediatric and Adolescent Gynecology, Philadelphia, Caderno, p.119-124, 2002.

INSTITUTO NACIONAL DE CÂNCER (Brasil). Diretrizes Brasileiras para o Rastreamento do Câncer do Colo do Útero. Rio de Janeiro: INCA, 2011. Disponível em: <http://www1.inca.gov.br/inca/Arquivos/Titulos/Nomenclatura_colo_do_utero.pd>. Acesso em: 6 jul. 2011.
JAKOBSSON, M.; GISSLER, M.; PAAVONEN, J.; TRAPPER, A. M. Loop electrosurgical excision procedure and the risk for preterm birth. Obstetrics and Gynecology, Chicago, Caderno, p.504-510, 2009.

LONGATTO FILHO, A.; ETILNGER, D.; GOMES, N. S.; CRUZ, S. V.; CAVALIERE, M. J. Frequency of abnormal uterine cervix smears from adolescents and adult women: review of 306,630 cases. Revista do Instituto Adolfo Lutz, São Paulo, n.62, p.31-34, 2003.

MCLAUGHLIN-DRUBIN, M. E.; MÜNGER, K. Oncogenic Activities of Human Papilloma viruses. Virus Research, Salamanca, Caderno, p.195-208, ago. 2009.

MOSCICKI, A. B. Conservative Management of Adolescents With Abnormal Cytology and Histology. Journal of the National Comprehensive Cancer Network, Washington, Caderno, p.101-106, jan. 2008.

MOSCICKI A.B., Ma Y et al. Risks for cervical intraepithelial neoplasia among adolescents and young women with abnormal cytology. Obstetrics and Gynecology, Chicago, Caderno, p.1335-1342, 2008.

MOSER, A. M.; REGGIANI, C.; URBANETZ, U. Comportamento sexual de risco entre estudantes universitárias dos cursos de ciências da saúde. Revista Associação Médica Brasileira, São Paulo, n.2, p.116-121, 2007.

PAIVA, V.; CALAZANS, G; VENTURI, G; DIAS, R. Idade e uso de preservativo na iniciação sexual de adolescentes brasileiros. Revista Saúde Pública, São Paulo, n. 42, p.45-53, fev. 2008.

PINTO, D. S; FUZII, H. T; QUARESMA, J. Á. S. Prevalência de infecção genital pelo HPV em populações urbana e rural da Amazônia Oriental Brasileira. Cadernos de Saúde Pública, Cidade, n.27, p.769-778, 2011.

ROBERTSON, Angela A.; LAWRENCE, Janet S. St.; MORSE, David T.; BAIRD-THOMAS, Connie; LIEW, Hui; GRESHAM, Kathleen. Comparison of Health Education 
and STD Risk Reduction Interventions for Incarcerated Adolescent Females. Health Education e Behavior, Mississippi, Caderno, p.241-250, 1 jun. 2011.

ROMERO, K. T.; MEDEIROS, E. H. G. R.; VITALLE, M. S. S.; WEHBA, J. O conhecimento das adolescentes sobre questões relacionadas ao sexo. Revista da Associação Médica Brasileira, n.53, p.14-19, 2007.

SELLORS, J. W; KARWALAJTYS, T. L; KACZOROWSKI, J.; MAHONY J. B.; LYTWYN, A.; CHONG, S.; et al. Incidence, clearance and predictors of human papillomavirus infection in women. Journal of Ayub Medical College, Abbottabad, Caderno, p.168-74, 18 fev. 2003.

SZWARCWALD, C. L.; BARBOSA-JÚNIOR, A.; PASCOM, A. R.; SOUZA-JÚNIOR, P. R. Knowledge, practices and behaviours related to HIV transmission among the Brazilian population in the 15-54 years age group, 2004. AIDS, Rockville, n.19, p.51-58, out. 2005.

Recebido em: 23 de Fevereiro de 2014 Avaliado em: 28 de Março de 2014 Aceito em: 14 de Julho de 2014
TARKOWSKI, T. A.; RAJEEVAN, M. S.; , LEE, D. R.; Unger E. R. Improved detection of viral RNA isolated from liquid-based cytology samples. The Journal of Molecular Diagnostics, Atlanta, Caderno, p.125-130, jun. 2001.

VENTURA, M.; IKAWA, D.; PIOVESAN, F.; BARSTED, L. $\mathrm{L}$. Direitos Sexuais e direitos reprodutivos na perspectiva dos direitos humanos: síntese para gestores, legisladores e operadores do Direito. Rio de Janeiro: ADVOCACI, 2003.

VITALLE, M. S. S. Alguns pontos conceituais sobre sexualidade na adolescência. Revista Paulista de Pediatria, São Paulo, n.2, p.89-93, jun. 2003.

WELLINGS, K.; COLLUMBIEN, M.; SLAYMAKER, E; SINGH, S.; HODGES, Z.; PATEL, D.; et al. Sexual behaviour in context: a global perspective. The Lancet, London, Caderno, p.1706-1728, 1 nov. 2006.

1. Médica formada pela Universidade Federal de Sergipe. Rua Cláudio Batista, s/n - Santo Antônio - Aracaju, Sergipe, Brasil. Email: meibarros83@gmail.com

2. Médica formada pela Universidade Federal de Sergipe. . Rua Cláudio Batista, s/n - Santo Antônio - Aracaju, Sergipe, Brasil. E- mail: jacquelinemazzotti@hotmail.com

3. Médico formado pela Universidade Federal de Sergipe. Rua Cláudio Batista, s/n - Santo Antônio - Aracaju, Sergipe, Brasil. E- mai: gtmatos82@ hotmail.com

4. Professora Adjunta da Disciplina de Ginecologia da Universidade Federal de Sergipe; PhD. Rua Cláudio Batista, s/n - Santo Antônio - Aracaju, Sergipe, Brasil. E-mail: julia.dias@globo.com 